\title{
The Observational Advantages of Euler Diagrams with Existential Import
}

\author{
Gem Stapleton $^{1}$, Atsushi Shimojima ${ }^{2}$, and Mateja Jamnik ${ }^{3}$ \\ 1 Centre for Secure, Intelligent and Usable Systems, University of Brighton, UK \\ g.e.stapleton@brighton.ac.uk \\ 2 Doshisha University, Japan \\ ashimoji@mail.doshisha.ac.jp \\ 3 Department of Computer Science and Technology, University of Cambridge, UK \\ mateja.jamnik@cl.cam.ac.uk
}

\begin{abstract}
The ability of diagrams to convey information effectively in part comes from their ability to make facts explicit that would otherwise need to be inferred. This type of advantage has often been referred to as a free ride and was deemed to occur only when a diagram was obtained by translating a symbolic representation of information. Recent work generalised free rides to the idea of an observational advantage, where the existence of such a translation is not required. Roughly speaking, it has been shown that Euler diagrams without existential import are observationally complete as compared to symbolic set theory. In this paper, we explore to what extent Euler diagrams with existential import are observationally complete with respect to set-theoretic sentences. We show that existential import significantly limits the cases when observational completeness arises, due to the potential for overspecificity.
\end{abstract}

\section{Introduction}

Diagrams are often seen as a useful tool in aiding our understanding of information, particularly in contrast to symbolic or textual notations. One of many reasons for this can be attributed to the ability of diagrams to convey facts in accessible manner, including facts that would otherwise need to be inferred from alternative representations. Such facts can be thought of as observable from the diagrammatic representation but inferrable from the alternative representation.

Previously, we introduced the theory of observational advantages [16], generalising the idea of a free ride [14]. In the case of free rides, one starts with a collection of statements, say $Q \subseteq P$ and $P \cap R=\emptyset$, and translates them into a semantically equivalent diagrammatic form, such as in Figure 1. The translation must ensure that the original statements can all be observed from the diagram. It can readily be seen, or observed, from Figure 1 that $Q \subseteq P$ (through curve containment) and $P \cap R=\emptyset$ (through curve disjointness). In addition, it can also be observed that $Q \cap R=\emptyset$, again through curve disjointness. This information, $Q \cap R=\emptyset$, is a free ride from the diagram given the original statements. In the case of observational advantages, the requirement for a translation is removed: 


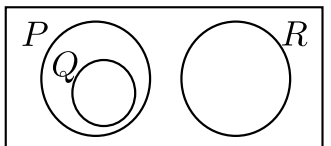

Fig. 1. No existential import and a free ride.

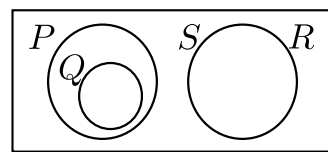

Fig. 2. Overspecificity issues.

the two representations must only be semantically equivalent, implying that all free rides are observational advantages but not vice versa.

The observational power of diagrams has long been recognised, with Hyperproof incorporating an observation-style inference rule in a proof system involving both diagrams and first-order logic [2]. Dretske's work, commonly described as "somebody's seeing that something is the case" [5], informed the development of the Euler/Venn inference system, where the authors called for the distinctive treatment of observation [18]. By formalising this insight into the benefit of diagrams, we can identify the set of statements that are observable, as opposed to inferrable, from a given statement.

In [16] we presented a formal framework for studying observational advantages and applied it to Euler diagrams (without existential import $[3]^{4}$ ) and set-theoretic sentences, limited to making subset and equality assertions. We proved that Euler diagrams were observationally complete: given any finite collection of set-theoretic sentences, $S$, from the class just described, there exists an Euler diagram, $d$, from which any set-theoretic sentence, $\sigma$, inferrable from $S$ can be observed. This is a significant result, because Euler diagrams are widely studied from the perspective of inference [4, 10,13], demonstrating that diagrams can be rich in observational advantages. An obvious question arises: if Euler diagrams are instead taken to have existential import, are they still rich in observational advantages? Given that many approaches exist to asserting non-emptiness (see [11]), including existential import, it is important to answer this question.

We extend our previous work to Euler diagrams with existential import and set-theoretic sentences which can also express the non-emptiness of a set and non-subset relations between sets. This allows us to show that there are restricted instances of when a finite set, $S$, of set-theoretic sentences has an equivalent Euler diagram, $d$, with existential import from which any set-theoretic sentence, $\sigma$, inferrable from $S$ can be observed. This is due to the overspecificity of Euler diagrams with existential import.

As an example, consider $Q \subseteq P, P \cap R=\emptyset, P \backslash Q \neq \emptyset, R=S$ and $Q \nsubseteq S$. One possible visualisation of this using Euler diagrams with existential import is in Figure 2. Clearly the four statements are represented in the diagram; focusing on $P \backslash Q \neq \emptyset$, this corresponds to the zone inside just the curve $P$ (so outside

\footnotetext{
${ }^{4}$ In Euler diagrams without existential import, zones can represent empty sets. By contrast, under existential import all zones in the diagram represent non-empty sets [7]. Peirce denotes non-emptiness of a set with $\otimes$-sequences [12] (also used by Shin [15] and further developed by Choudhury and Chakraborty [4]). Other notations use graphs to denote elements in sets $[6,8,9]$.
} 
$Q, R$ and $S$ ), which represents a non-empty set due to the existential import assumption now placed on the diagram's semantics. However, the diagram represents too much information such as $S \neq \emptyset$ : forcing zones to represent non-empty sets leads to this overspecificity problem $[14,17]$. Indeed, under the existential import semantics, there is no single Euler diagram that represents just these four statements and nothing more. In the rest of this paper, all Euler diagrams are assumed to be interpreted under the existential import semantics.

We provide the necessary and sufficient conditions under which a set of settheoretic sentences, formed using $\subseteq, \not$, = and $\neq$, can be visualised as an Euler diagram with existential import. We show that such a diagram is observationally complete. The conditions demonstrate that existential import may not only restrict the existence of an observationally complete diagram, but may prevent a semantically equivalent diagram to exist at all. Our results show that Euler diagrams with existential import suffer from overspecificity, which hugely limits their advantages over competing notations. This insight sets this paper apart from earlier work: it is the first to formally reveal that diagrams can have (substantial) limitations in exchange for the power to express a wider variety of information (such as $\not \subset$ and $\neq$ ). Consequently, designers and users of diagrams should pay careful attention when defining their syntax and semantics if one of their goals is to harness their observational power.

The paper is structured as follows. Section 2 discusses the idea of a meaningcarrying relationship and its role in observation. The syntax and semantics of Euler diagrams and the fragment of set theory that we consider are given in Section 3. We provide results on the model theory of these two systems in Sections 4 and 5 respectively, which are the necessary basis for understanding the limitations of Euler diagrams with existential import. Section 6 establishes the limited set of cases when observational completeness arises. We discuss these results and their implications in Section 7 and conclude in Section 8.

\section{Observation and Meaning-Carriers}

Central to the notion of observability is an understanding of how a representation of information conveys meaning through meaning-carrying relationships, which is discussed at some length in [16]. Here, due to space constraints, we provide a brief discussion along with various definitions from [16] that are essential for the remainder of this paper. As the context of this paper is on set theory and Euler diagrams, we provide examples from those domains.

A meaning-carrying relationship is a relation on the syntax of a statement that carries semantic value, evaluating to either true or false. This is similar to Shin's notion of a representing fact in her seminal work on Venn diagrams [15]. Meaning-carriers play an important role in both her work and ours. In settheoretic sentences, such as $P \subseteq Q$, there are single meaning-carrying relationships. In $P \subseteq Q$, the meaning-carrier is that $P$ is written to the left of $\subseteq$ and $Q$ to the right. Likewise, any set-theoretic statement formed using $\nsubseteq$, =, and 
$\neq$ has just a single meaning-carrier: the set written on the left has the asserted relationship with that written on the right.

A single Euler diagram can have numerous meaning-carrying relationships which are given by the spatial relationships between the curves. In Figure 2, the curves $S$ and $R$ are on top of one another, asserting that $S=R$. These meaningcarriers in the diagram give rise to the observable set-theoretic sentences. From Figure 2, we can observe $Q \subseteq P, Q \cap R=\emptyset$, and $S=R$, amongst many other things. Due to existential import, we can also observe $S \neq \emptyset$ and $P \backslash Q \neq \emptyset$.

One has to understand which syntactic relationships are meaning-carriers in order to define observability. In particular, one statement, $\sigma_{1}$, is observable from another, $\sigma_{2}$, if some meaning-carrying relationship in $\sigma_{2}$ corresponds directly to $\sigma_{1}$. For example, the containment of one curve, $Q$, by another, $P$, in an Euler diagram (Figure 2) is a meaning-carrier, allowing us to observe a subset relationship: $Q \subseteq P$. Likewise, when we have existential import, the presence of a region is a meaning-carrier: it represents a non-empty set. So, a set arising from a region, such as the zone inside $P$ and $Q$ in Figure 2, is non-empty: $P \cap Q \neq \emptyset$. We can also observe from the diagram $P \cap Q=Q$, since the region which represents $P \cap Q$ happens to be exactly the same region as that which represents $Q$.

Importantly, observability must respect semantics too: if a statement is observable then it must be semantically entailed (i.e., the observed statement must be true whenever the statement from which it is observed is true). We will define when a set-theoretic sentence can be observed from an Euler diagram later ${ }^{5}$. For now, we assume this definition is given and present a general definition of observability from a set of statements:

Definition 1. Let $\Sigma$ be a finite set of statements and $\sigma_{o}$ be a statement. Then $\sigma_{o}$ is observable from $\Sigma$ iff $\sigma_{o}$ is observable from some statement, $\sigma$, in $\Sigma$. The set of statements that are observable from $\Sigma$ is denoted $\mathcal{O}(\Sigma)[16]$.

We can now define what it means to be observationally complete:

Definition 2. Let $\Sigma$ and $\Sigma_{\vDash}$ be finite sets of statements. Then $\Sigma$ is observationally complete with respect to $\Sigma_{\models}$ if $\Sigma_{\models} \subseteq \mathcal{O}(\Sigma)[16]$.

Intuitively, the definition of observational completeness can be interpreted as follows: $\Sigma$ is a representation of information (such as a single diagram or a set of set-theoretic sentences) and $\Sigma_{\models}$ is a set of statements whose truth we wish to establish. If we can simply observe those statements to be true from $\Sigma$ then $\Sigma$ is observationally complete with respect to $\Sigma_{\models}$.

Definition 3. Let $\Sigma$ and $\hat{\Sigma}$ be finite, semantically equivalent sets of statements. Let $\sigma$ be a statement. If $\sigma$ is not observable from $\Sigma$ and $\sigma$ is observable from $\hat{\Sigma}$ then $\sigma$ is an observational advantage of $\hat{\Sigma}$ given $\Sigma$ [16].

Using the requirement that observable statements must be semantically entailed, we see that any statement, $\sigma$, which is an observational advantage of $\hat{\Sigma}$ given $\Sigma$ is semantically entailed by $\Sigma$.

\footnotetext{
${ }^{5}$ It is possible to define observability for other types of diagrams and statements too.
} 


\section{Set Theory and Euler Diagrams with Existential Import}

To develop the theory of observation and observational advantages in the case of set theory and Euler diagrams with existential import, we require a formalisation of both systems. Since a ready comparison of statements needs to be made across notations, the set of labels used to denote sets will be common to both set theory and Euler diagrams, as will their interpretation.

Definition 4. Define $\mathcal{L}$ to be a set whose elements are called labels. Two special symbols, $\emptyset$ and $U$, are not in $\mathcal{L}[16]$.

Definition 5. An interpretation is a pair, $\mathcal{I}=(\triangle, \Psi)$, where $\triangle$ is a set and $\Psi$ is a function, $\Psi: \mathcal{L} \cup\{\emptyset, U\} \rightarrow \mathbb{P} \triangle$, that maps labels to subsets of $\triangle$ and ensures that $\Psi(\emptyset)=\emptyset$ and $\Psi(U)=\triangle[16]$.

\subsection{Euler Diagrams with Existential Import}

We now introduce the syntax and semantics of Euler diagrams with existential import. The syntax remains unchanged from [16] and is included here for ease of reference. The semantics, however, differ due to the requirement that zones must represent non-empty sets. To begin, we formally define zones and regions.

Definition 6. A zone is a pair of finite, disjoint sets of labels, $\left(L_{i}, L_{o}\right)$, drawn from $\mathcal{L}$. A finite set of zones is a region.

In Figure 2, there are four zones. The zone inside just $P$ is $(\{P\},\{Q, R, S\})$ and the zone outside all the curves is $(\emptyset,\{P, Q, R, S\})$. This diagram uses four labels, so we write $L=\{P, Q, R, S\}$, where $L$ denotes the diagram's label set. The diagram's set of zones will be denoted $Z$, so in this case

$$
Z=\{(\{P\},\{Q, R, S\}),(\{P, Q\},\{R, S\}),(\{R, S\},\{P, Q\}),(\emptyset,\{P, Q, R, S\})\} .
$$

Formally, an Euler diagram is a set of labels together with a set of zones:

Definition 7. An Euler diagram, $d$, is a pair, $(L, Z)$, where $L$ is a finite subset of $\mathcal{L}$, and for all zones, $\left(L_{i}, L_{o}\right)$, in $Z$ it is the case that $L_{i} \cup L_{o}=L$. Given $d=(L, Z)$, we sometimes write $L(d)$ and $Z(d)$ for $L$ and $Z$ respectively. Given a finite set, $\mathcal{D}$, of Euler diagrams we define $L(\mathcal{D})$ to be $\bigcup_{d \in \mathcal{D}} L(d)$.

To define the semantics of Euler diagrams, it is useful to identify the zones that could be present in the diagram given the labels used, but which are in fact missing. Intuitively, missing zones represent the empty set.

Definition 8. Let $d=(L, Z)$ be an Euler diagram. The missing zones of $d$ are elements of $M Z(d)=\left\{\left(L_{i}, L \backslash L_{i}\right): L_{i} \subseteq L\right\} \backslash Z$.

We now extend the definition of an interpretation to identify the sets represented by zones and regions: 
Definition 9. Let $\mathcal{I}=(\triangle, \Psi)$ be an interpretation. An extension of $\Psi$ to map zones and regions to sets is defined as follows:

$$
\begin{aligned}
& \text { 1. for each zone, }\left(L_{i}, L_{o}\right), \Psi\left(L_{i}, L_{o}\right)=\bigcap_{l \in L_{i}} \Psi(l) \cap \bigcap_{l \in L_{o}} \overline{\Psi(l)} \text {, and } \\
& \text { 2. for each region, } r, \Psi(r)=\bigcup_{\left(L_{i}, L_{o}\right) \in r} \Psi\left(L_{i}, L_{o}\right) \text {. }
\end{aligned}
$$

Our next task is to define the circumstances under which an interpretation is a model for (i.e., agrees with the intuitive meaning of) an Euler diagram. As well as missing zones representing empty sets we also have to account for the existential import requirement: present zones represent non-empty sets:

Definition 10. Let $d=(L, Z)$ be an Euler diagram and $\mathcal{I}=(\triangle, \Psi)$ be an interpretation. Then $\mathcal{I}$ satisfies $d$ and is a model for d whenever $\Psi(z) \neq \emptyset$ for each zone $z$ in $Z$ and $\Psi(z)=\emptyset$ for each zone $z$ in $M Z(d)$.

\subsection{Set-Theoretic Sentences}

We now extend the work in [16] on set-theoretic sentences to allow statements to be made with $\neq$ and $\nsubseteq$, as well as $=$ and $\subseteq$. Firstly, we define set-theoretic expressions, which are syntactic representations of sets formed from the 'basic sets' represented by labels in $\mathcal{L}$ :

Definition 11. The following are set-theoretic expressions or, simply, setexpressions: (i) $U$ and $\emptyset$ are both set-expressions, (ii) every label in $\mathcal{L}$ is a setexpression, and (iii) if $s_{1}$ and $s_{2}$ are set-expressions then so are $\left(s_{1} \cap s_{2}\right),\left(s_{1} \cup s_{2}\right)$, $\left(s_{1} \backslash s_{2}\right)$, and $\overline{s_{1}}[16]$.

Given labels $P, Q$ and $R$, the following are some examples of set-theoretic expressions (omitting unnecessary brackets): $P, P \cap Q, Q \cup R, P \backslash(Q \cup R)$ and $\overline{(P \cap Q)}$. Often we will blur the distinction between syntax and semantics, talking of 'the set $P \cap Q$ ' when strictly speaking we mean the set represented by $P \cap Q$; given an interpretation, $(\triangle, \Psi)$, this set is $\Psi(P) \cap \Psi(Q)$. Set-theoretic expressions merely construct sets from the basic ones. We can then make assertions about the relationship between set-theoretic expressions using $\subseteq, \nsubseteq \subseteq$, and $\neq$ :

Definition 12. Given set-expressions $s_{1}$ and $s_{2}$ the following are set-theoretic sentences: $s_{1} \subseteq s_{2}, s_{1} \not \subseteq s_{2}, s_{1}=s_{2}$, and $s_{1} \neq s_{2}$. Sentences of the form $s_{1} \subseteq s_{2}$ and $s_{1}=s_{2}$ are positive whereas those of the form $s_{1} \nsubseteq s_{2}$ and $s_{1} \neq s_{2}$ are negative.

When we want to give set-expressions or set-theoretic sentences names, we will use $\equiv$. For example, to refer to $P \cap Q$ and $R \subseteq P \cap Q$ by the names $s_{1}$ and $s_{2}$, we write $s_{1} \equiv P \cap Q$ and $s_{2} \equiv R \subseteq P \cap Q$. This is to avoid overloading =. It is also helpful to us to have access to set of the labels, denoted $L(s)$, used in any given set-theoretic sentence, $s: L(s)$ is defined in the obvious recursive way. We extend this to a finite set, $\mathcal{S}$, of set-theoretic sentences: $L(\mathcal{S})$ is $\bigcup_{s \in \mathcal{S}} L(s)$, that is, the set of all labels appearing in members of $\mathcal{S}$. 
Now, to reiterate, every set-theoretic sentence only has one meaning-carrier: the set-expression on the left is in the asserted relationship with the set-expression on the right. This leads us to the definition of the semantics of set-theoretic sentences. The labels over which set-expressions are formed are already interpreted as sets (Definition 5). We extend this to cover the interpretation of more com-

plex set-expressions in order to identify when an interpretation 'agrees with' the intuitive meaning of (i.e., is a model for) sentences.

Definition 13. Let $\mathcal{I}=(\triangle, \Psi)$ be an interpretation. An extension of $\Psi$ to map set-expressions to sets is defined as follows. For each set-expression, $s$,

1. if $s \in \mathcal{L} \cup\{U, \emptyset\}$ then $\Psi(s)$ is already defined,

2. if $s \equiv\left(s_{1} \star s_{2}\right)$, where $\star \in\{\cap, \cup, \backslash\}$, then $\Psi(s)=\Psi\left(s_{1}\right) \star \Psi\left(s_{2}\right)$, and

3. if $s \equiv \overline{s_{1}}$ then $\Psi(s)=\overline{\Psi\left(s_{1}\right)}=\Psi(U) \backslash \Psi\left(s_{1}\right)$.

Definition 14. Let $s$ be a set-theoretic sentence. Let $\mathcal{I}=(\triangle, \Psi)$ be an interpretation. Then $\mathcal{I}$ satisfies $s$ and is a model for $s$ under the following circumstances:

1. if $s \equiv s_{1} \subseteq s_{2}$ then $\Psi\left(s_{1}\right) \subseteq \Psi\left(s_{2}\right)$,

2. if $s \equiv s_{1} \nsubseteq s_{2}$ then $\Psi\left(s_{1}\right) \nsubseteq \Psi\left(s_{2}\right)$,

3. if $s \equiv s_{1}=s_{2}$ then $\Psi\left(s_{1}\right)=\Psi\left(s_{2}\right)$, and

4. if $s \equiv s_{1} \neq s_{2}$ then $\Psi\left(s_{1}\right) \neq \Psi\left(s_{2}\right)$.

Let $\mathcal{S}$ be a finite set of set-theoretic sentences. Then $\mathcal{I}$ satisfies $\mathcal{S}$ and is a model for $\mathcal{S}$ provided $\mathcal{I}$ is a model for each set-theoretic sentence in $\mathcal{S}$.

\subsection{Semantic Relationships}

The final prerequisite for studying the observational advantages of Euler diagrams over set-theoretic sentences relies on us tying up their semantic relationships, beyond just mapping their (common) labels to sets in interpretations. We generically refer to Euler diagrams and set-theoretic sentences as statements.

Definition 15. Let $\sigma_{1}$ and $\sigma_{2}$ be statements. If $\sigma_{1}$ and $\sigma_{2}$ have the same models then they are semantically equivalent. If finite sets of statements, $\Sigma_{1}$ and $\Sigma_{2}$, have the same models then they are semantically equivalent.

Definition 16. Let $\Sigma$ be a finite set of statements and let $\sigma$ be a statement. Then $\Sigma$ semantically entails $\sigma$, denoted $\Sigma \vDash \sigma$, provided every model for $\Sigma$ is also a model for $\sigma$. If $\sigma$ is semantically entailed by, but not in $\Sigma$, then $\sigma$ is properly semantically entailed by $\Sigma$.

Lastly, since our focus is on the observational completeness of Euler diagrams with respect to set-theoretic sentences and the conditions under which this can be achieved, it is useful for us to introduce notation for the set of all set-theoretic sentences that are properly entailed given the labels used: 
Definition 17. Let $\mathcal{S}$ be a finite set of set-theoretic sentences. Define $\mathcal{S}_{\vDash}^{L(\mathcal{S})}$ to be the set of set-theoretic sentences that are properly semantically entailed by $\mathcal{S}$ such that each $s \in \mathcal{S}_{\vDash}^{L(\mathcal{S})}$ ensures $L(s) \subseteq L(\mathcal{S})$.

We can think of the labels used - that is, those in $L(\mathcal{S})$ - as being the sets of interest, since these are the sets about which $\mathcal{S}$ provides information. Then we can view $\mathcal{S}_{\models}^{L(\mathcal{S})}$ as containing precisely the set-theoretic sentences that make true statements about the sets of interest, but which are not explicitly given in $\mathcal{S}$. In other words, these are the statements that we can and must infer from $\mathcal{S}$.

\section{Model Theory: Euler Diagrams with Existential Import}

A major consideration for us is to identify when, given a set of set-theoretic sentences, $\mathcal{S}$, there exists a semantically equivalent Euler diagram, $d$. This is a prerequisite for identifying whether $d$ is observationally complete with respect to $\mathcal{S}$. Our strategy for this is to provide insight into what the models of Euler diagrams 'look like'. Unsurprisingly, this section establishes that the models for Euler diagrams with existential import are those for which all of the present zones represent non-empty sets and the missing zones represent empty sets. As it will be beneficial to us later, we define a relation on interpretations inspired by this insight:

Definition 18. Let $L \subseteq \mathcal{L}$ be a set of labels and $\mathcal{I}_{1}=\left(\triangle_{1}, \Psi_{1}\right)$ and $\mathcal{I}_{2}=$ $\left(\triangle_{2}, \Psi_{2}\right)$ be interpretations. Then $\mathcal{I}_{1}$ and $\mathcal{I}_{2}$ are $\boldsymbol{L}$-approximate, denoted $\mathcal{I}_{1} \approx_{L}$ $\mathcal{I}_{2}$, provided for every zone $\left(L_{i}, L_{o}\right)$ where $L_{i} \cup L_{o}=L, \Psi_{1}\left(L_{i}, L_{o}\right)=\emptyset$ iff $\Psi_{2}\left(L_{i}, L_{o}\right)=\emptyset$.

Intuitively, two interpretations are $L$-approximate if one never assigns the empty set to a zone formed over $L$ when the other does not. Clearly, $\approx_{L}$ is an equivalence relation on the set of interpretations.

Theorem 1. Let $d=(L, Z)$ be an Euler diagram. Then the set of models, $M(d)$, for $d$ is an equivalence class of interpretations under $\approx_{L}$. In particular, $\mathcal{I}=$ $(\triangle, \Psi)$ is in $M(d)$ iff for each zone, $z_{p}$, in $Z(d)$, we have $\Psi\left(z_{p}\right) \neq \emptyset$ and for each zone, $z_{m}$, in $M Z(d)$ we have $\Psi\left(z_{p}\right)=\emptyset$.

Theorem 1 demonstrates the highly constrained nature of models for Euler diagrams with existential import: they are single equivalence classes of $L$ approximate interpretations, forcing present zones to represent non-empty sets. There is no possibility for representing uncertainty when it comes to the nonemptiness of a set. By contrast, if the existential import requirement is removed (so a present zone can be empty or not) then the model sets are unions of equivalence classes: the models are given by $M_{Z_{1}} \cup \ldots \cup M_{Z_{2^{n}}}$ where: $Z_{1}$ to $Z_{2^{n}}$ are the $2^{n}$ subsets of $Z$, and $M_{Z_{i}}$ is the equivalence class of interpretations where for each zone, $z_{p}$ in $Z_{i}$, we have $\Psi\left(z_{p}\right) \neq \emptyset$ and for each zone, $z_{m}$ in $\left(Z(d) \backslash Z_{i}\right) \cup M Z(d)$ we have $\Psi\left(z_{p}\right)=\emptyset$. 


\section{Model Theory: Set-Theoretic Sentences}

In order to identify when a set of set-theoretic sentences, $\mathcal{S}$, has a semantically equivalent diagram, $d$, we start by appealing to Theorem 1 . This theorem tells us that $\mathcal{S}$ only has such a diagram if its models are also a single equivalence class under $\approx_{L}$. Clearly, such an equivalence class determines a set of zones that represent (non)empty sets. It is therefore useful to introduce the idea of determining set-emptiness:

Definition 19. Let $\mathcal{S}$ be a finite set of set-theoretic sentences. We say $\mathcal{S}$ determines set-emptiness if the set of models, $M(\mathcal{S})$, for $\mathcal{S}$ forms an equivalence class of interpretations under $\approx_{L(\mathcal{S})}$.

For example, consider the following:

$$
\begin{aligned}
& \mathcal{S}_{1}=\{P \nsubseteq Q, Q \nsubseteq P\}, \\
& \mathcal{S}_{2}=\{P \nsubseteq Q, Q \nsubseteq P, P \cap Q=\emptyset\}, \text { and } \\
& \mathcal{S}_{3}=\{P \nsubseteq Q, Q \nsubseteq P, P \cap Q=\emptyset, \overline{P \cup Q} \neq \emptyset\} .
\end{aligned}
$$

Note $L\left(\mathcal{S}_{1}\right)=L\left(\mathcal{S}_{2}\right)=L\left(\mathcal{S}_{3}\right)=\{P, Q\}$. Among these, only $\mathcal{S}_{3}$ determines set-emptiness. $\mathcal{S}_{1}$ does not, since it can be satisfied by both, an interpretation assigning the empty set to zone $(\{P, Q\},\{\})$, and one assigning a non-empty set to it, for example. These interpretations are not $L\left(\mathcal{S}_{1}\right)$-approximate, so $\mathcal{S}_{1}$ does not determine set-emptiness. With the addition of sentence $P \cap Q=\emptyset$, the set of models for $\mathcal{S}_{2}$ no longer has interpretations that 'disagree' on the zone $(\{P, Q\}, \emptyset)$ (every model assigns the empty set to it), yet models can still disagree on zone $(\emptyset,\{P, Q\})$. Adding another sentence, $\overline{P \cup Q} \neq \emptyset$, to give $\mathcal{S}_{3}$ makes all models agree on the zones that can be formed over $\{P, Q\}$ : they assign the empty set to $(\emptyset,\{P, Q\})$ and non-empty sets to $(\{P\},\{Q\}),(\{Q\},\{P\})$, and $(\emptyset,\{P, Q\}))$; a semantically equivalent Euler diagram is given in Figure 3. Thus, determining set-emptiness is rather a high demand to place on the case of settheoretic sentences: only very limited sets of set-theoretic sentences determine set-emptiness. By contrast, determining set-emptiness is not placing such a high demand on Euler diagrams. Indeed, every single Euler diagram determines setemptiness. We obtain the following lemma:

Lemma 1. Let $\mathcal{S}$ be a set of set-theoretic sentences. Then $\mathcal{S}$ has a semantically equivalent Euler diagram only if $\mathcal{S}$ determines set-emptiness.

This lemma indicates the extent of the overspecificity of Euler diagrams, relative to set-theoretic sentences. The phenomenon of overspecificity has been pointed out in [17] and further investigated in [14] in connection to a wider variety of diagrams. However, the impact of the phenomenon in a specific diagrammatic system has never been formalised. Our approach illustrates how it can be investigated.

Our next goal is to characterise the sets of set-theoretic sentences that meet the demand of determining set-emptiness. To begin, we notice that positive settheoretic sentences provide information about the emptiness of sets; $P \subseteq Q$ tells 


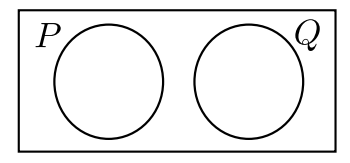

Fig. 3. Translating set-theoretic sentences.

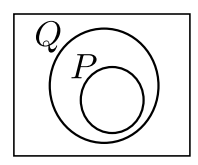

Fig. 4. Relevant zones.

us that $P \backslash Q=\emptyset$ and $R=S$ tells us that $R \backslash S=\emptyset$ and $S \backslash R=\emptyset$. Moreover, negative set-theoretic sentences provide information about the non-emptiness of sets; $P \nsubseteq Q$ expresses $P \backslash Q \neq \emptyset$ and $R \neq S$ implies $R \backslash S \neq \emptyset$ or $S \backslash R \neq \emptyset$. It is therefore useful to distinguish the positive and negative cases:

Definition 20. Given a set of set-theoretic sentences $\mathcal{S}$, we define $\mathcal{S}^{+}$and $\mathcal{S}^{-}$ to be the set of all positive and negative members of $\mathcal{S}$, respectively.

So, positive sentences provide information about empty zones whereas negative sentences provide information about non-empty zones. This leads to the idea of a relevant zone, which relies on a translation of a set-theoretic sentence to a region which is determined by the sets of interest. For example, given $L=\{P, Q\}$ as the sets of interest, the expression $P \backslash Q$ corresponds to the zone $(\{P\},\{Q\})$ since, informally, $(\{P\},\{Q\})$ represents the set of things in $P$ that are not in $Q$, that is, $P \backslash Q$. Likewise, the expression $P$ - again given $L=\{P, Q\}$ - corresponds to the region $\{(\{P\},\{Q\}),(\{P, Q\}, \emptyset)\}$ : the elements in $P$ can be either in $P \backslash Q$, corresponding to $(\{P\},\{Q\})$, or in $P \cap Q$, corresponding to $(\{P, Q\}, \emptyset)$.

Definition 21. Let $s$ be a set-expression and let $L$ be a set of labels such that $L(s) \subseteq L$. The translation of $s$ given $L$ into a region, denoted $\mathcal{T}(s, L)$, is defined recursively:

1. if $s \equiv \emptyset$ then $\mathcal{T}(s, L)=\emptyset$,

2. if $s \equiv U$ then $\mathcal{T}(s, L)=\left\{\left(L_{i}, L_{o}\right): L_{i} \cup L_{o}=L \wedge L_{i} \cap L_{o}=\emptyset\right\}$,

3. if $s \in \mathcal{L}$ then $\mathcal{T}(s, L)=\left\{\left(L_{i}, L_{o}\right) \in \mathcal{T}(U, L): s \in L_{i}\right\}$,

4. if $s \equiv\left(s_{1} \star s_{2}\right)$, where $\star \in\{\cap, \cup, \backslash\}$, then $\mathcal{T}(s, L)=\left(\mathcal{T}\left(s_{1}, L\right) \star \mathcal{T}\left(s_{2}, L\right)\right)$, and

5. if $s \equiv \overline{s_{1}}$ then $\mathcal{T}(s, L)=\left(\mathcal{T}(U, L) \backslash \mathcal{T}\left(s_{1}, L\right)\right)$.

Using the translation of set-expressions to regions, we can now see how to translate set-theoretic sentences to regions too. For instance, $P \subseteq Q$ is true whenever $\Psi(P) \subseteq \Psi(Q)$. In terms of zones formed over $P$ and $Q$, the sentence $P \subseteq Q$ is true whenever

$$
\Psi(\{(\{P\},\{Q\}),(\{P, Q\}, \emptyset)\}) \subseteq \Psi((\{Q\},\{P\}),(\{P, Q\}, \emptyset)) .
$$

Figure 4 illustrates $P \subseteq Q$ and we see that the zone $(\{P\},\{Q\})$ is missing. Therefore $\left(^{*}\right)$ is true, and the zone $(\{P\},\{Q\})$ is relevant in this case.

Definition 22. Given a set-theoretic sentence $s$ and a set of labels $L$ such that $L(s) \subseteq L$, we define the relevant set of zones of $s$ given $L$, denoted $\mathcal{R} \mathcal{Z}(s, L)$, in the following way: 
1. If $s$ is of the form $s_{1}=s_{2}$ or $s_{1} \neq s_{2}, \mathcal{R Z}(s, L)=\left(\mathcal{T}\left(s_{1}, L\right) \backslash \mathcal{T}\left(s_{2}, L\right)\right) \cup$ $\left(\mathcal{T}\left(s_{2}, L\right) \backslash \mathcal{T}\left(s_{1}, L\right)\right)$

2. If $s$ is of the form $s_{1} \subseteq s_{2}$ or $\left.s_{1} \nsubseteq s_{2}, \mathcal{R Z}(s, L)=\mathcal{T}\left(s_{1}, L\right) \backslash \mathcal{T}\left(s_{2}, L\right)\right)$.

So, continuing with the example above, we have

$$
\mathcal{R Z}(P \subseteq Q,\{P, Q\})=\{(\{P\},\{Q\})\}
$$

and, whenever $P \subseteq Q$, we know that $(\{P\},\{Q\})$ represents the empty set. So, the relevant set of zones of a set-theoretic sentence, $s$, is 'relevant' to $s$ in that the zones help to determine when $s$ is satisfied by an interpretation. The following lemma makes this point more precise.

Lemma 2. Let $s$ be a set-theoretic sentence and $\mathcal{I}=(\triangle, \Psi)$ be an interpretation. Let $L$ be a set of labels such that $L(s) \subseteq L$. Then

1. if $s$ is positive then $\mathcal{I}$ is a model for $s$ iff $\Psi(\mathcal{R Z}(s, L))=\emptyset$,

2. if $s$ is negative then $\mathcal{I}$ is a model for $s$ iff $\Psi(\mathcal{R Z}(s, L)) \neq \emptyset$ for some zone $z \in \mathcal{R Z}(s, L)$.

Our next goal is to identify conditions under which any set of set-theoretic sentences, $\mathcal{S}$, determines set-emptiness. To produce such conditions, it is important to have an understanding of what the models for $\mathcal{S}$ 'look like'. We can gain such insight by considering the models for $\mathcal{S}^{+}$and $\mathcal{S}^{-}$separately, informed by Lemma 2, noting that the models for $\mathcal{S}$ must model both $\mathcal{S}^{-}$and $\mathcal{S}^{+}$.

The set of relevant zones, in the case of positive set-theoretic sentences, gives us information about which zones must represent the empty set. In this sense, the positive set-theoretic sentences in $\mathcal{S}$ partially characterise the models for $\mathcal{S}$. By Lemma 2, an interpretation, $\mathcal{I}=(\triangle, \Psi)$, is in $M\left(\mathcal{S}^{+}\right)$(the set of models for $\left.\mathcal{S}^{+}\right)$iff, for each $s$ in $\mathcal{S}^{+}, \Psi(\mathcal{R Z}(s, L(\mathcal{S}))=\emptyset$. Therefore, $\mathcal{I}=(\triangle, \Psi)$ is in $M\left(\mathcal{S}^{+}\right)$provided

$$
\bigcup_{s \in \mathcal{S}^{+}} \Psi(\mathcal{R Z}(s, L(\mathcal{S}))=\emptyset .
$$

For ease of notation, we define the empty zones of $\mathcal{S}$ to be elements of

$$
\mathcal{E} \mathcal{Z}(\mathcal{S})=\bigcup_{s \in \mathcal{S}^{+}} \mathcal{R Z}(s, L(\mathcal{S}))
$$

and the Venn zones of $\mathcal{S}$ to be elements of

$$
\mathcal{V Z}(\mathcal{S})=\left\{\left(L_{i}, L(\mathcal{S}) \backslash L_{i}\right): L_{i} \subseteq L(\mathcal{S})\right\} .
$$

The empty zones represent empty sets in all models for $\mathcal{S}$. The remaining zones in the Venn zone set may or may not represent empty sets. Now, we have some information about non-emptiness, provided by $\mathcal{S}^{-}$, but it need not completely determine whether any given zone is necessarily non-empty in a model. This is where $\mathcal{S}^{-}$must be considered carefully. 
From Lemma 2, we know that an interpretation, $\mathcal{I}=(\triangle, \Psi)$, is in $M\left(\mathcal{S}^{-}\right)$iff, for each $s^{-}$in $\mathcal{S}^{-}, \Psi(z) \neq \emptyset$ for some $z \in \mathcal{R} \mathcal{Z}\left(s^{-}, L(\mathcal{S})\right)$. For $\mathcal{S}$ to determine setemptiness, therefore, we seek conditions on $\mathcal{S}^{-}$that are necessary and sufficient to ensure that each zone in $\mathcal{V} \mathcal{Z}(\mathcal{S}) \backslash \mathcal{E}(\mathcal{S})$ represents a non-empty set.

In this context, we aim to identify sets of zones that partially characterise some of the models for $\mathcal{S}^{-}$: given a set of zones, $Z$, under what conditions is the set of interpretations that map the zones in $Z$ to non-empty sets a set of models for $\mathcal{S}^{-}$? As a first step, we introduce the idea of a choice function, which assigns relevant zones to negative set-theoretic sentences. Importantly, assigned zones cannot be empty zones.

Definition 23. Let $\mathcal{S}$ be a finite set of set-theoretic sentences. A choice function, $c$ : $\mathcal{S}^{-} \rightarrow \mathcal{V} \mathcal{Z}(\mathcal{S}) \backslash \mathcal{E} \mathcal{Z}(\mathcal{S})$ for $\mathcal{S}$, maps negative set-theoretic sentences in $\mathcal{S}$ to zones such that for each $s^{-} \in \mathcal{S}^{-}, c\left(s^{-}\right) \in \mathcal{R} \mathcal{Z}\left(s^{-}, L(\mathcal{S})\right)$.

Clearly, given an arbitrary $\mathcal{S}$ there need not exist a choice function. This occurs when there is a negative set-theoretic sentence in $\mathcal{S}$ such that all of its relevant zones are in $\mathcal{E} \mathcal{Z}(\mathcal{S})$. Under such circumstances, it is obvious that $\mathcal{S}$ has no models and is, therefore, inconsistent. However, given an arbitrary choice function, $c$, the zones in $\mathcal{V Z}(\mathcal{S}) \backslash \mathcal{E} \mathcal{Z}(\mathcal{S})$ to which $c$ maps set-theoretic sentences (i.e., the set of zones that is the image of $c$ ) partially characterises some of the models for $\mathcal{S}^{-}$: all interpretations where these zones represent non-empty sets are models for $\mathcal{S}^{-}$. Intuitively, any given model for $\mathcal{S}^{-}$is classified by some choice function.

So far, we have characterised all of the models for $\mathcal{S}^{+}$and the models for $\mathcal{S}^{-}$. In a build-up to our set of necessary and sufficient conditions that identify when $\mathcal{S}$ defines set-emptiness, we establish when $\mathcal{S}$ is satisfiable, using choice functions. We start by building an interpretation using a choice function.

Definition 24. Let $\mathcal{S}$ be a finite set of set-theoretic sentences for which there exists a choice function, $c: \mathcal{S}^{-} \rightarrow \mathcal{V Z}(\mathcal{S}) \backslash \mathcal{E} \mathcal{Z}(\mathcal{S})$. We define the choice interpretation for $\mathcal{S}$ given $c$ to be the interpretation $\mathcal{I}_{\mathcal{S}}^{c}=(\triangle, \Psi)$ as follows:

1. the universal set, $\triangle$, is the image of $c$, that is:

$$
\triangle=\left\{z \in \mathcal{V} \mathcal{Z}(\mathcal{S}) \backslash \mathcal{E} \mathcal{Z}(\mathcal{S}): \exists s^{-} \in \mathcal{S}^{-} c\left(s^{-}\right)=z\right\}, \quad \text { and }
$$

2. for each $l \in \mathcal{L}$, we define

$$
\Psi(l)= \begin{cases}\left\{\left(L_{i}, L_{o}\right) \in \triangle: l \in L_{i}\right\} & \text { if } l \in L(\mathcal{S}) \\ \emptyset & \text { otherwise. }\end{cases}
$$

Lemma 3 establishes that the choice interpretation is a model for $\mathcal{S}$ :

Lemma 3. Let $\mathcal{S}$ be a finite set of set-theoretic sentences for which there exists a choice function, $c: \mathcal{S}^{-} \rightarrow \mathcal{V Z}(\mathcal{S}) \backslash \mathcal{E} \mathcal{Z}(\mathcal{S})$. The choice interpretation, $\mathcal{I}_{\mathcal{S}}^{c}=$ $(\triangle, \Psi)$, for $\mathcal{S}$ given $c$ is a model for $\mathcal{S}$. 
Lemma 3 builds on our insight into what sets of models 'look like' for $\mathcal{S}$. We have seen that choice functions can be used to define models. Importantly, the absence of a choice function implies the absence of models: $\mathcal{S}$ is unsatisfiable.

Choice functions with different images correspond to models that are not $L(\mathcal{S})$-approximate. In particular, if there is a non-surjective choice function then there are necessarily models for $\mathcal{S}$ that are not $L(\mathcal{S})$-approximate. This semantic intuition is captured syntactically via choice functions in Theorem 2 .

Theorem 2. Let $\mathcal{S}$ be a finite set of set-theoretic sentences. Then $\mathcal{S}$ determines set-emptiness iff there exists a choice function for $\mathcal{S}$ and all choice functions for $\mathcal{S}$ are surjective.

Thus, Theorem 2 is what is needed to meet our major goal for this section: the provision of necessary and sufficient conditions for determining set-emptiness. The models for such an $\mathcal{S}$ are characterised by the following theorem:

Theorem 3. Let $\mathcal{S}$ be a finite set of set-theoretic sentences that determines setemptiness. Let $\mathcal{I}=(\triangle, \Psi)$ be an interpretation. Then $\mathcal{I}$ is a model for $\mathcal{S}$ iff

1. the empty zones of $\mathcal{S}$ all represent the empty set: $\Psi(\mathcal{E Z}(\mathcal{S}))=\emptyset$, and

2. the remaining zones all represent non-empty sets: for all $z \in \mathcal{V} \mathcal{Z}(\mathcal{S}) \backslash \mathcal{E} \mathcal{Z}(\mathcal{S})$, $\Psi(z) \neq \emptyset$.

\section{Observational Completeness}

We now set out to identify an Euler diagram that is observationally complete given a set-emptiness defining $\mathcal{S}$. Focusing first on the requisite Euler diagram, $d$, for $\mathcal{S}$, we need $d$ to have the same models as $S$. That is, $d$ 's present zones (which represent non-empty sets) should correspond to $\mathcal{V Z}(\mathcal{S}) \backslash \mathcal{E} \mathcal{Z}(\mathcal{S})$, since these are precisely the zones that represent non-empty sets in all models for $\mathcal{S}$. Likewise, the zones not in $d$ should correspond to those in $\mathcal{E} \mathcal{Z}(\mathcal{S})$, since these represent empty sets in all models for $\mathcal{S}$. We have already seen an example of the Euler diagram for a given set of set-theoretic sentences in Figure 3, given $\mathcal{S}_{3}$ on page 9.

Definition 25. Let $\mathcal{S}$ be a finite set of set-theoretic sentences that determines set-emptiness. The Euler diagram for $\mathcal{S}$, denoted $d_{\mathcal{S}}$, is

$$
d_{\mathcal{S}}=(L(\mathcal{S}), \mathcal{V} \mathcal{Z}(\mathcal{S}) \backslash \mathcal{E} \mathcal{Z}(\mathcal{S})) .
$$

Importantly, $\mathcal{S}$ and $d_{\mathcal{S}}$ are semantically equivalent, which follows from Theorems 1 and 3 :

Theorem 4. Let $\mathcal{S}$ be a finite set of set-theoretic sentences that determines setemptiness. Then $\mathcal{S}$ and $d_{\mathcal{S}}$ are semantically equivalent.

We must now consider what it means for a sentence to be observable from an Euler diagram, generalising [16]. To do this, we need to translate regions to set-expressions. Intuitively, regions translate to multiple set-expressions. For 
instance, in Figure 4, the region comprising the single zone inside the curve $P$ corresponds to various set-expressions, including $P$ and $P \cap Q$, since this zone represents both the set $P$ and the set $P \cap Q$; indeed, in this case $P=P \cap Q$. For our purposes here it is sufficient to have an intuitive understanding of what set-expressions can arise from regions, along the lines of the example just given ${ }^{6}$. Using this intuitive approach, we can now define observability:

Definition 26. Let $d$ be an Euler diagram and let $s \equiv s_{1} \star s_{2}$, where $\star \epsilon$ $\{\subseteq, \nsubseteq,=, \neq\}$, be a set-theoretic sentence. Then $s \equiv s_{1} \star s_{2}$ is observable from $d$ provided there exist regions $r_{1}$ and $r_{2}$ of $d$ such that

1. $r_{1} \star r_{2}$,

2. $s_{1}$ is a translation of $r_{1}$, and

3. $s_{2}$ is a translation of $r_{2}$.

Finally, we have one of our key results:

Theorem 5. Let $\mathcal{S}$ be a finite set of set-theoretic sentences that determines setemptiness. Then $\left\{d_{\mathcal{S}}\right\}$ is observationally complete with respect to $\mathcal{S}_{\vDash}^{L(\mathcal{S})}$.

\section{Discussion}

Our results on Euler diagrams with existential import demonstrate that there are severe limitations due to overspecificity, at least from the perspective of observational advantages. This is potentially problematic since diagrams, by their very nature, are believed to excel as representations of information due to their ability to make facts explicit that would otherwise need to be inferred.

To recap, an Euler diagram, $d$, with existential import is only semantically equivalent to a finite set, $\mathcal{S}$, of set-theoretic sentences when $\mathcal{S}$ determines setemptiness. This is a serious limitation, arising because the models for $d$ are a single equivalence class under the $L$-approximate relation. The crux of the problem is that such diagrams require complete certainty over whether zones represent empty sets. By contrast, most sets of set-theoretic sentences do not make this demand on their model sets and are, in this case, more expressive than their diagrammatic counterpart.

This suggests that diagrams which allow uncertainty to be expressed, and thus avoid overspecificity, are more likely to have observational advantages over competing notations. Indeed, suppose that the existential import requirement is removed and, instead, Peirce's $\otimes$-sequences are used to express non-emptiness. We conjecture that any finite set, $\mathcal{S}$, of set-theoretic sentences (as in Definition 12) will be semantically equivalent to some diagram, $d$. Moreover, we expect $d$ to be observationally complete with respect to $\mathcal{S}_{\models}^{L(\mathcal{S})}$. Such diagrams do not suffer from overspecificity issues and have models that are unions of equivalence classes under the $L$-approximate relation, just like sets of set-theoretic sentences.

${ }^{6}$ It is straightforward, yet lengthy, to define a translation from regions to setexpressions; due to space constraints, we refer the reader to [16]. 

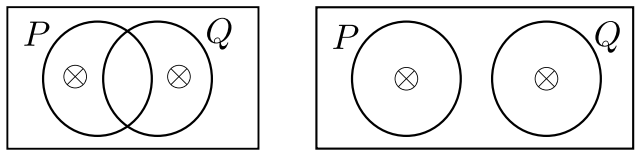

Fig. 5. Exploiting $\otimes$-sequences to overcome overspecificity limitations.

To illustrate, consider again $\mathcal{S}_{1}=\{P \nsubseteq Q, Q \nsubseteq P\}$. Whilst no Euler diagram with existential import can express this information, we could use an Euler diagram with $\otimes$-sequences instead to define non-emptiness (left of Figure 5). From the diagram, we can observe, for instance, that $P \backslash Q \neq \emptyset$ due to $\otimes$ inside $P$ but outside $Q$. The diagram on the right of Figure 5 illustrates how we can depict $\mathcal{S}_{2}=\{P \nsubseteq Q, Q \nsubseteq P, P \cap Q=\emptyset\}$. It will be interesting to extend the work in this paper to determine whether this alternative system of Euler diagrams is indeed observationally complete for any given $\mathcal{S}$. Importantly, in this alternative system, the zones containing no $\otimes$ symbol can represent either empty or nonempty sets, thus removing the overspecificity arising from existential import.

\section{Conclusion}

The ideas of observation, observational advantages and observational completeness enable us to formally compare different representations of information. It is considered advantageous if a representation of information simply allows us to observe other statements of interest to be true. Therefore, this suggests that designing notations that allow many observations to be made, especially compared to competing representations, is sensible. In the case of diagrams, free rides and observational advantages are seen as a major feature that indicates how and when they may be more efficacious than symbolic or textual notations.

We demonstrated that overspecificity makes diagrams less observationally advantageous. As in the case of Euler diagrams with existential import, overspecificity often means there is no corresponding diagram for a given representation of information. This is clearly undesirable and leads us to posit that diagrams should be carefully designed in order to ensure that they do not have overspecifity issues and also support the observability of information. Indeed, our results indicate an advantage of set-theoretic language: it can express information freely, whether the information is strong enough to determine set-emptiness or not. Euler diagrams with existential import are disadvantageous in that respect.

There is still much work to be done, however, to ascertain the extent to which observational advantages are also cognitive advantages. We think it is important to understand the net cognitive value of observability. There is certainly cognitive cost associated with observing statements, but to what extent is this cost 'lower' than the alternative task of inferring information instead? The net cognitive value of a statement observable from a diagram depends on the cost of recognising a meaning-carrying relationship and also on the set of available operations to translate this meaning-carrier into an alternative representation. 
This research needs to be, in the future, connected to a psychological and computational model of the perceptual operations available to people alongside the formal investigations that we have begun. Preliminary work in [1] is exploring this important cognitive aspect, and it will be interesting to see how it develops.

Acknowledgements Stapleton and Jamnik were funded by a Leverhulme Trust Research Project Grant (RPG- 2016-082) for the project entitled Accessible Reasoning with Diagrams.

\section{References}

1. Baigelenov, A., M, S., Hung, Y.H., Parsons, P.: Toward an understanding of observational advantages in information visualization. In: IEEE Conference on Information Visualization, Poster Abstracts (2017)

2. Barwise, J., Etchemendy, J.: Hyperproof. CSLI Press (1994)

3. Chatti, S., Schang, F.: The cube, the square and the problem of existential import. History and Philosophy of Logic 34(2), 101-132 (2013)

4. Choudhury, L., Chakraborty, M.K.: On extending Venn diagrams by augmenting names of individuals. In: 3rd International Conference on the Theory and Application of Diagrams. LNAI, vol. 2980, pp. 142-146. Springer (2004)

5. Dretske, F.: Seeing and Knowing. Routledge \& Kegan Paul, London, UK (1969)

6. Gil, J., Howse, J., Kent, S.: Formalising spider diagrams. In: IEEE Symposium on Visual Languages. pp. 130-137. IEEE (1999)

7. Hammer, E., Shin, S.J.: Euler's visual logic. History and Philosophy of Logic pp. $1-29$ (1998)

8. Howse, J., Stapleton, G., Taylor., J.: Spider diagrams. LMS Journal of Computation and Mathematics 8, 145-194 (2005)

9. Kent, S.: Constraint diagrams: Visualizing invariants in object oriented models. In: Proceedings of OOPSLA97. pp. 327-341. ACM Press (October 1997)

10. Mineshima, K., Okada, M., Takemura, R.: A diagrammatic inference system with Euler circles. Journal of Logic, Language and Information 21(3), 365-391 (2012)

11. Moktefi, A., Pietarinen, A.V.: On the diagrammatic representation of existenial statements with Venn diagrams. Journal of Logic, Language and Information 24(4), 361-374 (2015)

12. Peirce., C.: Collected Papers, vol. 4. Harvard University Press (1933)

13. Sato, Y., Mineshima, K., Takemura, R.: The Efficacy of Euler and Venn Diagrams in Deductive Reasoning: Empirical Findings. In: 6th International Conference on the Theory and Application of Diagrams. pp. 6-22. Springer (2010)

14. Shimojima, A.: Semantic Properties of Diagrams and Their Cognitive Potentials. CSLI (2015)

15. Shin, S.J.: The Logical Status of Diagrams. Cambridge University Press (1994)

16. Stapleton, G., Jamnik, M., Shimojima, A.: What makes an effective representation of information: A formal account of observational advantages. Journal of Logic, Language and Information 26(2), 143-177 (2017)

17. Stenning, K., Oberlander, J.: A cognitive theory of graphical and linguistic reasoning: Logic and implementation. Cognitive Science 19(1), 97-140 (1995)

18. Swoboda, N., Allwein, G.: Using DAG transformations to verify Euler/Venn homogeneous and Euler/Venn FOL heterogeneous rules of inference. Journal on Software and System Modeling 3(2), 136-149 (2004) 